\title{
Folk knowledge of wild food plants among the tribal communities of Thakht- e-Sulaiman Hills, North-West Pakistan
}

\author{
Khalid Ahmad ${ }^{1 *}$ and Andrea Pieroni ${ }^{2}$
}

\begin{abstract}
Background: Indigenous communities of the Thakht-e-Sulamian hills reside in the North-West tribal belt of Pakistan, where disadvantaged socio-economic frames, lack of agricultural land and food insecurity represent crucial problems to their survival. Several studies in diverse areas worldwide have pointed out the importance of wild food plants (WFPs) for assuring food sovereignty and food security, and therefore the current study was aimed at documenting traditional knowledge of WFPs and analyzing how this varies among generations.

Methods: Ethnobotanical data were collected during 2010-2012. In total of seventy-two informants were interviewed in ten villages via in-depth interviews and group discussions with key informants followed by freelisting. Data were analyzed through descriptive and inferential statistics and novelty was checked by comparing the gathered data with the published literature.

Results: A total of fifty-one WFP species belonging to twenty-eight families were documented. Rosaceae was the dominant family with the largest number of species and highest frequency of citation (FC). July was the peak month for availability of WFPs, and fruit was the most commonly consumed part. Among the most cited species, Olea ferrugenia was ranked first with a $F C=1$, followed by Amaranthus spinosus ( $F C=0.93)$. Of the documented species about $14 \%$ (7) were marketable and $27 \%$ (14) were reported for the first time to be used as WFP species in Pakistan.

Conclusion: WFPs still play an important role in the food and culture of the study area and the folk knowledge attached to them is remarkable in the region, although declining among the younger generations. The recorded species needs to be re-evaluated in local projects aimed at fostering endogenous strategies of food security, as well as re-evaluating cultural heritage and sustaining small-scale food market circuits.
\end{abstract}

Keywords: Wild Food Plants, Ethnobotany, Thakht-e-Sulaiman Hills, Pakistan

\section{Background}

A large variety of wild plants are used as food in diverse communities around the globe. These WFPs vary with the surrounding biodiversity and consequently influence food habits [1]. Wild food flora is a vital element of the diet of rural populations. It suits the community of that particular belief and culture as a food because of its traditionally acquired knowledge-based principles, feelings, and manners. Wild collected food plants have been part of the human diet since time immemorial and it has

\footnotetext{
*Correspondence: khalid.taxonomist@gmail.com

'Department of Environmental Sciences, COMSATS Institute of Information

Technology, Abbottabad, Pakistan

Full list of author information is available at the end of the article
}

been argued that past societies made more use of wild flora than is done nowadays [2,3]. Due to their remarkable nutrient values as well as being an excellent source of minerals, fiber, vitamins, and fatty acids that add flavor and color to the diet, WFPs play a key role in complementing staple foods [4-7].

Despite the marked increase in food production, $33 \%$ of mountain people in developing countries are facing hunger, malnutrition and starvation [8]. By the year 2050 the world's population is expected to reach 9 billion, requiring $70-100 \%$ more food than today. This issue demands substantial attention in order to explore new food assets. The genetic manipulation of crops is considered a potential way to enhance quantity and 
protect them from diseases and various stresses [9]. WFPs have considerable potential for the development of new crops through domestication and provide a genetic reserve for hybridization and selection [10, 11]. Furthermore, previous ethnobotanical surveys indicate that more than 7000 species are being used by humans as food and for livelihood in poor communities [12, 13].

At present, human populations rely on a small number of cultivated species, domesticated during the past 13,000 years that partly reduced both the beneficial and toxic effects of secondary plant metabolites [14, 15]. In contrast, the human genome cannot fully adjust to the intense changes in diet and lifestyle, which is still adapted to the pre-agriculturalist diet pattern. Modern lifestyle diseases are due to alterations in major functional dietary components which were endowed with prophylactic effects in wild gathered food [3, 15-19]. Some important WFP knowledge is restricted to particular communities but such specific knowledge decreases quite rapidly due to its fragile nature. Therefore, ethnobotanical field research for documentation and evaluation of this traditional knowledge is of great importance [20] in providing insight into food diversification which must be identified.

Pakistan is a developing country with an area of about 10 million hectares $(0-8611 \mathrm{~m}$ altitude), which has different climatic zones-tropical, subtropical and temperate -featuring a unique and vast floral diversity that harbors more than 6000 vascular plant species [21-23]. Despite the various climatic zones and floral diversity, Pakistan is ranked $11^{\text {th }}$ in the Food Security Risk Index [24]. An alarming situation is presented by the National Nutrition Survey of Pakistan [25] which stated that almost 58 \% households are food insecure. Furthermore, the WHO reported in 2010 that $50 \%$ of child deaths are directly or indirectly due to malnutrition in Pakistan [26]. The availability of WFPs in different seasons becomes more important when cultivated fruits and vegetables are not available.

In spite of their great importance, WFPs are vanishing from traditional diets, which poses serious concerns due to their role and contribution in the cultural history of a region as well as their nutraceutical value [27]. In the developing world these plants are regularly ignored in governmental policies, agricultural research and extension programs. Over the past decade, the majority of tribal communities on the north-western boarder of Pakistan have been affected by the 'war on terror', which has destabilized their traditional knowledge systems. The present research area is semi-arid and mountainous with deficient agricultural land. The people live in extreme poverty with widespread food insecurity. They are also not considered in government developmental policies.
The present study focused on these remote indigenous communities of the Thakht-e-Sulaiman hills, NW Pakistan, to document their knowledge and practices concerning WFPs, which have not been previously explored. The specific aims of this study were:

- to document folk knowledge regarding WFPs in the study area;

- to determine how this knowledge is distributed between locations at lower and higher elevations and among generations in the study area;

- to compare the collected data with the overall Pakistani ethnobotanical literature, in order to possibly identify novel wild food plant records.

\section{Methods}

Study area and ethnic background

The Sulaiman mountain range is a geologically important area which forms a border between the Iranian Plateau and the Asian subcontinent. As the highest Plateau in the west and southwest, this range acts as a natural barrier to humid winds coming from the Indian Ocean thus creating arid conditions across southern Afghanistan. Centrally, the Sulaiman range lies a little east of $70^{\circ} \mathrm{E}$ longitude, and it is geologically composed of great folds of the Cretaceous series. The top of the mountain range is called Thakht-e-Sulaiman-locally known as Kaesagher. The present study was based on the ethnobotanical analysis of WFPs present on the eastern side of the Thakht-e-sulaiman Hills (Fig. 1). This area is situated in the north-west (NW) of Pakistan and serves as a border between the Frontier Region of Dera Ismail Khan (F.R, D.I.Khan) and the Zhobe District of Baluchistan Province. In particular, the study area is encompassed by South Waziristan Agency (north), D.I. Khan (east), Dera Ghazi Khan (south) and Zhob (west). The area is a small administrative unit under Federally

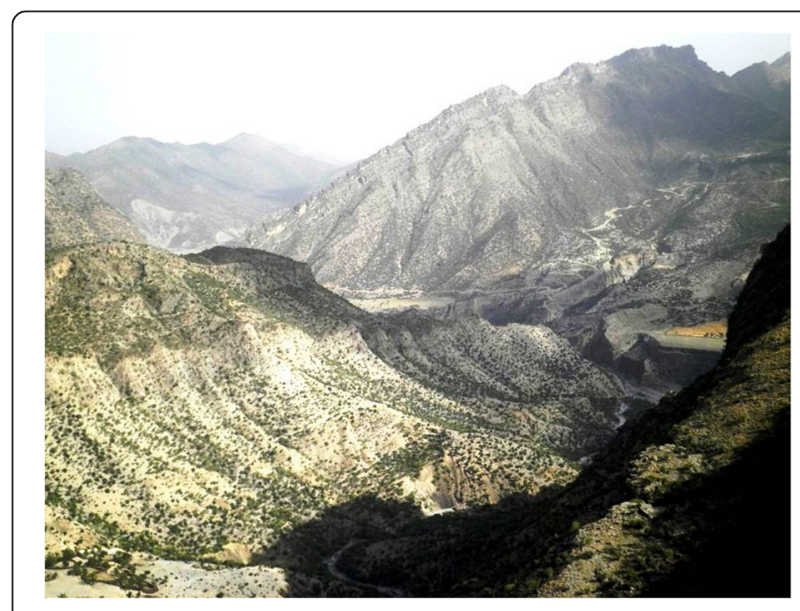

Fig. 1 Landscape of the study area 
Administered Tribal Areas (FATA) of Pakistan (Fig. 2) and two tribes, Sherani and Ustranas, inhabit this frontier region [28]. The Sherani area is totally under the eastern shadow of the highest peak of Thakht-eSulaiman. These mountains are covered by coniferous forest and exhibit arid to semi-arid lands that receive 200-500 mm of bimodal precipitation annually [29]. The region experiences hot summers (June, July and August) in which the temperature reaches $40{ }^{\circ} \mathrm{C}$ with a monthly rainfall of $21-33 \mathrm{~mm}$ and cold winters (December, January and February) in the range of 5.7$7.6{ }^{\circ} \mathrm{C}$ with a monthly rainfall of $13 \mathrm{~mm}$ [30].

\section{Field methods}

Ethnobotanical field work was conducted for a period of two years during 2010-2012. The study area was divided in two portions based on their altitude: we defined as foothill villages those villages located between 750 and 1,800 m.a.s.l., while mountain/migratory villages were those villages located between 1,800 and 3,000 m.a.s.l. A total of seventy-two informants (sixty -two men and ten women) from ten different villages were interviewed on the traditional uses of WFPs (including wild fruits, wild vegetables and wild tea species). The ages of the interviewees ranged from 20 to 90 years with an average of $41.8 \pm 14.5$ years. Detailed unstructured and semistructured, formal and informal interviews were performed with key informants and group discussions were conducted during the first phase of the study [31]. The first phase was designed to help understand the general perceptions of the locals about natural phenomena [32], to become familiar with ethnographic terms and their emic definitions, and in particular to learn about their interactions with local flora. In the second phase of data collection, successive 'oral freelisting' sessions were performed to obtain more salient species and to check disparities in individual and village-wise knowledge. Detailed structured interviews were mostly followed by freelisting. We provided supplementary prompting to enlarge the freelisting [33]. Special care was taken to avoid non-genuine information [34, 35] and responses were cross-checked through informal methods for confirmation. Consent was taken from each informant before every interview and the objectives and procedures

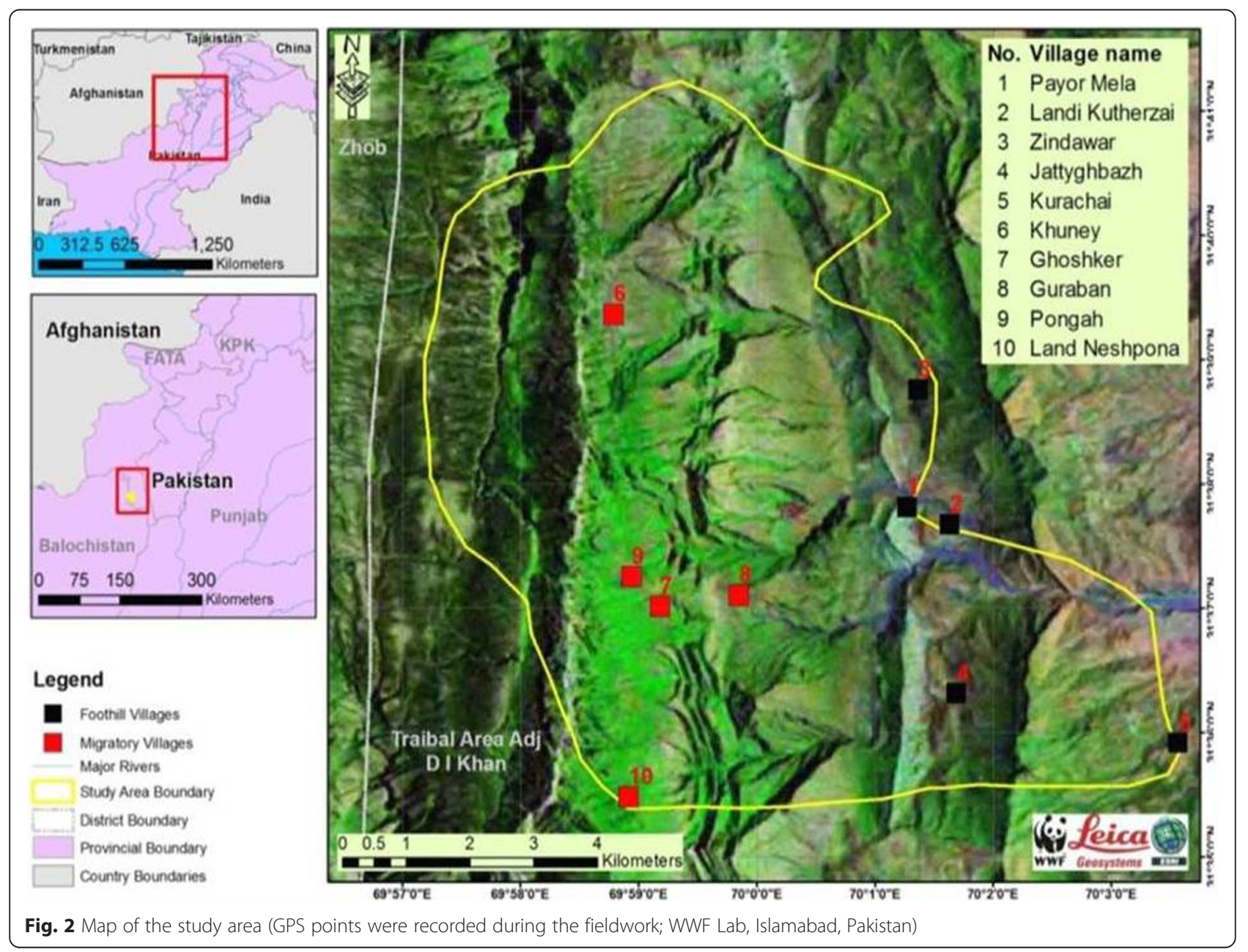


of the project were clearly explained. The local language (Pashtu) was used to conduct the interviews with informants, which the first author was able to properly understand. We maintained a continuing relationship with the local communities in order to develop a sense of trust and to be allowed to stay within the foothill and migratory villages and to accompany the locals during their daily activities and to participate in their ceremonies. National and international laws, especially the rules of the Convention on Biological Diversity (CBD) and the ethical guidelines of the International Society of Ethnobiology [36] were strictly followed.

\section{Sample}

An informant from nearly every $3^{\text {rd }}$ house was included in the sample (interview) with minor variations. The population and demographic details of the five foothill villages are shown in Table 1. The locals of two foothill villages (i.e. Payor Mela and Landi Kutherzai) migrate to the high mountains in summer. So, the mountain/migratory villages were based on the residents from these foothill villages while other inhabitants of those villages were from low altitude areas (not included in the study). The names, altitude, and number of houses of each foothill and mountain/migratory village are given in Table 2.

\section{Collection and identification of plant specimens}

Plant specimens were collected in triplicate according to standard botanical and ethnobotanical protocols [37]. The specimens were identified by taxonomists at Quaidi-Azam University, Islamabad, Pakistan and confirmed by matching their taxonomic characters with the Flora of Pakistan [38, 39]. Family names were assigned according to the Angiosperm Phylogeny Group [40] while species names were assigned in accordance with 'The Plant List' [41]. Voucher specimens were numbered and deposited in the Herbarium of Pakistan (ISL) for future reference work.

\section{Data analysis}

Freelisting data were analyzed through descriptive statistics to determine more salient species and informant consensus, using Excel spreadsheets. To check the effect of variables on diversity and variation in folk plant knowledge of individuals and villages, pivot tables were used. T-tests were performed to examine the significance of differences among different categories, while MannWhitney-U-Tests for independent samples were performed to establish the distribution of FC values among different groups. FC was determined by counting the number of informants that cited the use of the species divided by the total number of informants that participated in the study. To compare the present data with the published literature from the country, information was gathered from online sources using Google Scholar and Web of Sciences as search engines.

\section{Results and discussion}

\section{Documentation of wild food plants}

Ethnobotanical data of WFPs including vernacular names, parts used, gathering period, gathering areas, collectors and folk food uses are reported in Table 3. A total of fifty-one WFPs from forty-two genera and 28 families were documented. The family Rosaceae was dominant with the largest number of species (7 spp.), followed by Asteraceae (4 spp.), while Apocynaceae, Malvaceae and Rhamnaceae were represented by 3 species each. Based on FC values, the dominant family was Rosaceae followed by Oleaceae, whereas the remaining families with high FC values included Pinaceae, Alliaceae and Moraceae. Most of these taxa are widespread and typically used as wild foods, which have been previously reported in different Eurasian countries [42-46]. However, a rather uncommon food taxon is Apocynaceae- a family known for its toxicity and medicinal potential. Olea ferrugenia, Amaranthus spinosus, and Ficus palmata exhibited the highest FC values (1.0, 0.93 and 0.69, respectively), whereas in other species $\mathrm{FC}$ ranged from

Table 1 Social characteristics of the sample and study sites

\begin{tabular}{|c|c|c|c|c|c|}
\hline Village name & PayorMela & LandiKutherzai & Zindawar & JattyGhbaz & Kurachai \\
\hline Village size (number of families) & 36 & 55 & 21 & 42 & 25 \\
\hline Sample size(number of informants) & 18 & 22 & 5 & 18 & 9 \\
\hline $\begin{array}{l}\text { Dependency on livestock as a source } \\
\text { of income }\end{array}$ & $75 \%$ & $64 \%$ & $87 \%$ & $55 \%$ & $67 \%$ \\
\hline Informants average age & $48.0 \pm 18.6$ & $40.9 \pm 13.7$ & $36.0 \pm 6.5$ & $41.3 \pm 12.6$ & $36.7 \pm 12.2$ \\
\hline Average number of family members & $14.9 \pm 5.6$ & $12.4 \pm 6.6$ & $15.6 \pm 6.3$ & $14.7 \pm 6.2$ & $10.0 \pm 2.4$ \\
\hline${ }^{a}$ Migration ratio & $50 \%$ (to mountains) & $61 \%$ (to mountains) & $50 \%$ (within foothills) & $38 \%$ (within foothills) & 0 \\
\hline Bilingualism & $54 \%$ & $25 \%$ & $1 \%$ & $24 \%$ & $8 \%$ \\
\hline Average/month/head expenditure (in PKR) & $931 \pm 212$ & $1063 \pm 370$ & $941 \pm 194$ & $1166 \pm 537$ & $1420 \pm 457$ \\
\hline
\end{tabular}

${ }^{(a)}$ : in two villages people migrate to the mountains, while in two other villages inhabitants migrate within the foothills area during the summer season; a migration ratio of $50 \%$ indicates that half of the families of a village migrate in summer 
Table 2 Geographic characteristics of the study sites

\begin{tabular}{|c|c|c|c|}
\hline Village name & $\begin{array}{l}\text { Elevation } \\
\text { (m.a.s.l.) }\end{array}$ & $\begin{array}{l}\text { Number of } \\
\text { households }\end{array}$ & Soil, area, population, and vegetation \\
\hline PayorMela & 1116 & 36 & $\begin{array}{l}\text { The village has stony soil and thin vegetation within the immediate surroundings, but there is a reserved } \\
\text { forest and a very fertile valley in the vicinity; population and area of the village are moderate in size. }\end{array}$ \\
\hline LandiKutherzai & 1052 & 55 & $\begin{array}{l}\text { The village contains fertile loamy soil that can support dense and diverse vegetation; it has a large } \\
\text { population and a small area that make the surroundings of the village nearly barren; there are no reserve } \\
\text { forests nearby. }\end{array}$ \\
\hline Zindawar & 1146 & 21 & $\begin{array}{l}\text { The village contains various types of soil and a diverse flora. It has a small population and a large area; the } \\
\text { vegetation is dense and comparatively undisturbed; it also exhibits floral elements of higher altitudes } \\
\text { (mountains). }\end{array}$ \\
\hline JattyGhbaz & 1155 & 45 & $\begin{array}{l}\text { The village contains stony soil; the village population is moderately sized but the area is large; the vegetation } \\
\text { is relatively dense. }\end{array}$ \\
\hline Kurachai & 1184 & 25 & $\begin{array}{l}\text { The village has mostly stony soil, which cannot support herbaceous flora; it has a restricted area and a } \\
\text { modest population; the vegetation is under anthropogenic pressure. }\end{array}$ \\
\hline Khuney & 2384 & 16 & $\begin{array}{l}\text { It exhibits various types of soils and a diverse flora; the village has a large area and a small population; the } \\
\text { density and diversity of the surrounding vegetation is moderate. }\end{array}$ \\
\hline Goraban & 2456 & 26 & $\begin{array}{l}\text { The village contains fertile loamy soil; it has a small area and a modest population; the village is located in a } \\
\text { type of valley, which shows diverse vegetations. }\end{array}$ \\
\hline Ghoshker & 2301 & 37 & $\begin{array}{l}\text { The village contains mostly stony soil, which cannot support herbaceous flora. It has a moderate area but a } \\
\text { large population; the surrounding vegetation is not dense and is under anthropogenic pressure. }\end{array}$ \\
\hline Pongah & 2562 & 40 & $\begin{array}{l}\text { The village possesses various types of soils which supports a diverse flora; it has a modest area but a large } \\
\text { population; the surrounding vegetation is moderately dense and diverse. }\end{array}$ \\
\hline $\begin{array}{l}\text { Land } \\
\text { Nishpona }\end{array}$ & 2584 & 22 & $\begin{array}{l}\text { The village contains loamy soil and a diverse and dense flora; it has a small population and a large area; the } \\
\text { surrounding vegetation is dense and undisturbed. }\end{array}$ \\
\hline
\end{tabular}

(Note: The first five villages are foothill villages while the last five villages are mountain/migratory villages)

0.59 to 0.01 . These and related species have been reported by multiple authors from different parts of the country [42, 47-54]. Fruits were the most commonly consumed plant parts (31 spp.) followed by leaves (12 spp.), a finding similar to earlier studies [11, 42]. Regarding growth habit, herbs were dominant (43\%) followed by shrubs, trees and climbers $(31,20$ and $6 \%$ respectively). The number of species for each growth habit were significantly different, but the FC values of tree species were the highest followed by shrubs.

\section{Wild food plant categories}

The WFPs were categorized into three groups, i.e. wild fruits (56\%); wild vegetables (33\%) and wild tea (11\%). In the study area, wild fruits were mostly collected by boys below 25 years of age. It has been previously reported that fruits are a highly consumed food category among WFPs $[20,55,56]$. Wild fruits serve as an alternative to cultivated food during dry seasons and times of famine. In most rural communities of developing countries, wild fruits are the only fruits which are consumed by the local people as they cannot afford to buy commercial fruits [57]. In the present study, the number of wild vegetable species was less compared to other ethnobotanical studies [54, 58]. This might be attributed to less annual rainfall [28], insufficient ingredients used in cooking, and excessive use of dairy products among local communities. Similarly, in the study area, the use of wild vegetables was common among communities residing in the foothills, whereas those with large herd sizes migrated to the mountains and preferred dairy products. The lesser importance of wild vegetables in pastoral communities has also been reported by [59]. Although the use of wild tea species was presently uncommon among local inhabitants, their use was documented as this knowledge is vanishing. Inhabitants of the area use 6 species as alternative teas when commercial teas are unavailable or found only at great distances from their settlements.

\section{Diversity and variation in folk plant knowledge}

In the study area, the transfer of WFP knowledge was from parents to children (vertical) as well as within the community (horizontal), but weaker contacts were developed among different villages. In total, 47, 40, 36, 24 and 24 plant species were documented in Landi Kutherzai, Payor Mela, Jatty Ghbaz, Zindawar and Kurachai villages, respectively. Of these, 31 \% (16 spp.) were known to all foothill village informants, while $18 \%$ (9 spp.) were only known in a single village. Among the remaining plant species, 24, 12 and $16 \%$ were common to 4,3 and 2 villages respectively. Village size, exposure to different ecological zones (seasonal migration-see Table 1) and surrounding flora (See Table 2) may contribute to this variation. For example, Landi Kutherzai and Payor Mela 
Table 3 Folk uses of wild food plants recorded in the study area

\begin{tabular}{|c|c|c|c|c|c|c|c|c|}
\hline $\begin{array}{l}\text { Botanical taxon, voucher specimen } \\
\text { code and botanical family }\end{array}$ & Folk name & $\begin{array}{l}\text { Used } \\
\text { part(s) }\end{array}$ & $\begin{array}{l}\text { Gathering } \\
\text { period }\end{array}$ & $\begin{array}{l}\text { Gathering } \\
\text { area(s) }\end{array}$ & Collectors & Folk food uses & $\begin{array}{l}\text { Citation } \\
\text { frequency }\end{array}$ & $\begin{array}{l}\text { Previously } \\
\text { reported } \\
\text { from Pakistan } \\
\text { as WFPs }\end{array}$ \\
\hline $\begin{array}{l}\text { Aerva javanica (Burm.f.) Juss. } \\
\text { ex Schult. (127216) }\end{array}$ & Sparokai & Leaves & April-July & Waste land & Women & \multirow{2}{*}{$\begin{array}{l}\text { Young fresh leaves are boiled in water for } 1 / 2 \text { hour. The extra } \\
\text { water is poured out, and the leaves are moved to another } \\
\text { container containing ghee with fried onions \& red chilies. } \\
\text { Kept on the fire until water is evaporated \& ghee appears } \\
\text { on top. This is then eaten with bread. }\end{array}$} & \multirow[t]{2}{*}{0.16} & \multirow[t]{2}{*}{ Yes } \\
\hline Amaranthaceae & & & & & & & & \\
\hline Allium carolinianum DC. (127194) & \multirow[t]{2}{*}{ Khokhai } & \multirow[t]{2}{*}{ Whole plant } & \multirow{2}{*}{$\begin{array}{l}\text { June- } \\
\text { September }\end{array}$} & \multirow[t]{2}{*}{ Ridges } & \multirow[t]{2}{*}{ Men } & \multirow[t]{2}{*}{ The leaves and bulbs are eaten with bread. } & \multirow[t]{2}{*}{0.37} & \multirow[t]{2}{*}{ Yes } \\
\hline Amaryllidaceae & & & & & & & & \\
\hline Allium sp. Linn. (127217) & \multirow[t]{2}{*}{ Cook } & \multirow[t]{2}{*}{ Whole plant } & \multirow{2}{*}{$\begin{array}{l}\text { June- } \\
\text { September }\end{array}$} & \multirow{2}{*}{$\begin{array}{l}\text { Slopes and } \\
\text { ridges }\end{array}$} & \multirow[t]{2}{*}{ Men } & \multirow{2}{*}{$\begin{array}{l}\text { The leaves and bulbs are eaten with bread and are also } \\
\text { used as spice in curry. }\end{array}$} & \multirow[t]{2}{*}{0.50} & \multirow[t]{2}{*}{ Yes } \\
\hline Amaryllidaceae & & & & & & & & \\
\hline Amaranthus spinosus L. (127219) & \multirow[t]{2}{*}{ Sarmay } & \multirow[t]{2}{*}{ Leaves } & \multirow[t]{2}{*}{ April-July } & \multirow[t]{2}{*}{ Waste land } & \multirow[t]{2}{*}{ Women } & \multirow{2}{*}{$\begin{array}{l}\text { Young fresh leaves are boiled in water for } 1 / 2 \text { hour. The } \\
\text { extra water is poured out, and then the leaves are moved } \\
\text { to another container containing ghee with fried onions \& } \\
\text { red chilies. Kept on the fire until water is evaporated \& } \\
\text { ghee appears on top. This is then eaten with bread. }\end{array}$} & \multirow[t]{2}{*}{0.93} & \multirow[t]{2}{*}{ Yes } \\
\hline Amaranthaceae & & & & & & & & \\
\hline $\begin{array}{l}\text { Berberis calliobotrys Bien. ex } \\
\text { Koehne (127222) }\end{array}$ & Sarmay & Fruits & October & Pine forests & Men & Directly consumed. & 0.11 & No \\
\hline \multicolumn{9}{|l|}{ Berberidaceae } \\
\hline $\begin{array}{l}\text { Caragana ambigua Stocks } \\
(127205)\end{array}$ & \multirow[t]{2}{*}{ Zaray } & \multirow[t]{2}{*}{ Flowers } & \multirow[t]{2}{*}{ June } & \multirow[t]{2}{*}{ Valleys } & Kids & Directly consumed. & 0.02 & No \\
\hline Fabaceae & & & & & & & & \\
\hline $\begin{array}{l}\text { Caralluma tuberculata N.E.Br. } \\
\text { (127133) }\end{array}$ & Pamanai & Stems & March-April & $\begin{array}{l}\text { Moist shady } \\
\text { places }\end{array}$ & $\begin{array}{l}\text { Women, } \\
\text { men, kids }\end{array}$ & $\begin{array}{l}\text { Extensive salt is rubbed on the cut pieces and left for } \\
\text { half an hour. They are then washed with water. Now, }\end{array}$ & 0.22 & Yes \\
\hline Apocynaceae & & & & & & $\begin{array}{l}\text { they are fried in hot boiling ghee containing fried onions. } \\
\text { This is then eaten with bread. }\end{array}$ & & \\
\hline Celtis australis L. (127176) & Thaghah & Fruits & May & Near human & Men, kids & Directly consumed. & 0.05 & Yes \\
\hline Cannabaceae & & & & & & & & \\
\hline Cicer nuristanicum Kitam. (127183) & Chenrah & Fruit & July & High mountain & Kids & Directly consumed. & 0.06 & No \\
\hline Fabaceae & & & & & & & & \\
\hline Cirsium arvense (L.) Scop. (127231) & Da khwarak & Stems & May-August & Waste land & Men, kids & The ectoderm of the semi-mature stem is removed. & 0.06 & No \\
\hline Asteraceae & & & & & & & & \\
\hline $\begin{array}{l}\text { Cotoneaster microphyllus Wall. Ex } \\
\text { Lindl. (127234) }\end{array}$ & Manray & Fruit & May & $\begin{array}{l}\text { Bushy } \\
\text { vegetation }\end{array}$ & Men, kids & Directly consumed. & 0.30 & Yes \\
\hline Rosaceae & & & & & & & & \\
\hline Cotoneaster minutus Klotz (127125) & Sharavo & Fruit & September & Shrubby & Men, kids & Directly consumed. & 0.59 & Yes \\
\hline Rosaceae & & & & $\begin{array}{l}\text { mountain } \\
\text { vegetation }\end{array}$ & & & & \\
\hline
\end{tabular}


Table 3 Folk uses of wild food plants recorded in the study area (Continued)

\begin{tabular}{|c|c|c|c|c|c|c|c|c|}
\hline $\begin{array}{l}\text { Cotoneaster pruinosus Klotz } \\
\text { (127206) }\end{array}$ & Pushthawergai & Fruit & August & \multirow[t]{2}{*}{$\begin{array}{l}\text { Beside } \\
\text { pedestrian } \\
\text { passes }\end{array}$} & \multirow[t]{2}{*}{ Kids } & \multirow[t]{2}{*}{ Directly consumed. } & \multirow[t]{2}{*}{0.22} & \multirow[t]{2}{*}{ No } \\
\hline Rosaceae & & & & & & & & \\
\hline $\begin{array}{l}\text { Cynoglossum lanceolatum Forssk. } \\
\text { (127212) }\end{array}$ & \multirow[t]{2}{*}{ Jezgai } & \multirow[t]{2}{*}{ Leaves } & \multirow[t]{2}{*}{ April-July } & \multirow[t]{2}{*}{ Waste land } & \multirow[t]{2}{*}{ Women } & \multirow{2}{*}{$\begin{array}{l}\text { Young fresh leaves are boiled in water for } 1 / 2 \text { hour. The } \\
\text { extra water is poured out and then the leaves are moved } \\
\text { to another container containing ghee with fried onions \& } \\
\text { red chilies. Kept on the fire until water is evaporated \& } \\
\text { ghee appears on top. This is then eaten with bread. }\end{array}$} & \multirow[t]{2}{*}{0.01} & \multirow[t]{2}{*}{ No } \\
\hline Boraginaceae & & & & & & & & \\
\hline $\begin{array}{l}\text { Debregeasia saeneb (Forssk.) } \\
\text { Hepper \& J.R.I.Wood (127143) }\end{array}$ & Mermandai & Fruit & May & $\begin{array}{l}\text { High mountain } \\
\text { valleys }\end{array}$ & Men, kids & Directly consumed. & 0.19 & Yes \\
\hline \multicolumn{9}{|l|}{ Urticaceae } \\
\hline Ficus palmata Forssk. (127240) & \multirow[t]{2}{*}{ Injar } & \multirow[t]{2}{*}{ Leaves, Fruit } & \multirow{2}{*}{$\begin{array}{l}\text { April } \\
\text { (Leaves), July } \\
\text { (Fruit) }\end{array}$} & \multirow{2}{*}{$\begin{array}{l}\text { Near human } \\
\text { settlements }\end{array}$} & \multirow{2}{*}{$\begin{array}{l}\text { Women, } \\
\text { men, kids }\end{array}$} & \multirow{2}{*}{$\begin{array}{l}\text { The fruits are directly consumed. The young fresh leaves } \\
\text { are boiled in water for } 2 \text { hours to soften completely. This } \\
\text { is then eaten directly or sometimes with bread. }\end{array}$} & \multirow[t]{2}{*}{0.69} & \multirow[t]{2}{*}{ Yes } \\
\hline Moraceae & & & & & & & & \\
\hline $\begin{array}{l}\text { Grewia tenax (Forssk.) Fiori } \\
(127126)\end{array}$ & Pasthawnai & Fruit & August & Hedgerows & Kids & Directly consumed. & 0.01 & Yes \\
\hline \multicolumn{9}{|l|}{ Malvaceae } \\
\hline Grewia villosa Willd. (127243) & \multirow[t]{2}{*}{ Injarai } & \multirow[t]{2}{*}{ Fruit } & \multirow[t]{2}{*}{ July } & \multirow{2}{*}{$\begin{array}{l}\text { Marshy \& bushy } \\
\text { vegetation }\end{array}$} & \multirow[t]{2}{*}{ Kids } & \multirow[t]{2}{*}{ Directly consumed. } & \multirow[t]{2}{*}{0.08} & \multirow[t]{2}{*}{ Yes } \\
\hline Malvaceae & & & & & & & & \\
\hline Lactuca dissecta D.Don (127184) & \multirow[t]{2}{*}{ Paywerka } & \multirow[t]{2}{*}{ Leaves } & April-August & Hedgerows & Kids & Directly consumed. & 0.05 & No \\
\hline Asteraceae & & & & & & & & \\
\hline $\begin{array}{l}\text { Launaea procumbens (Roxb.) } \\
\text { Ramayya \& Rajagopal (127180) }\end{array}$ & Sondrashi & Leaves & April-August & Waste land & Men, Kids & Directly consumed. & 0.12 & Yes \\
\hline Asteraceae & & & & & & & & \\
\hline $\begin{array}{l}\text { Limonium cabulicum (Boiss.) } \\
\text { Kuntze (127170) }\end{array}$ & Botyarai & Leaves & June-August & $\begin{array}{l}\text { Rocky barren } \\
\text { land }\end{array}$ & Men & $\begin{array}{l}\text { The fresh leaves are boiled in water containing sugar. } \\
\text { This is then taken as tea. }\end{array}$ & 0.05 & No \\
\hline Plumbaginaceae & & & & & & & & \\
\hline Malva sylvestris L. (127123) & Methrai & Leaves & April-July & Waste land & Women & Young fresh leaves are boiled in water for $1 / 2$ hour. The & 0.08 & Yes \\
\hline Malvaceae & & & & & & $\begin{array}{l}\text { moved to another container containing ghee with } \\
\text { fried onions \& red chilies. Kept on the fire until water } \\
\text { is evaporated \& ghee appears on top. This is then } \\
\text { eaten with bread. }\end{array}$ & & \\
\hline $\begin{array}{l}\text { Notholirion thomsonianum } \\
\text { (Royle) Stapf (127166) }\end{array}$ & Shyajey & Leaves & April-May & $\begin{array}{l}\text { Sandy fertile } \\
\text { places }\end{array}$ & $\begin{array}{l}\text { Women, } \\
\text { men }\end{array}$ & $\begin{array}{l}\text { Young fresh leaves are boiled in water for } 1 / 2 \text { hour. } \\
\text { The extra water is poured out, and then the leaves }\end{array}$ & 0.31 & No \\
\hline Liliaceae & & & & & & $\begin{array}{l}\text { with fried onions \& red chilies. Kept on the fire until } \\
\text { water is evaporated \& ghee appears on top. This is } \\
\text { then eaten with bread. }\end{array}$ & & \\
\hline $\begin{array}{l}\text { Olea ferruginea Wall. ex Aitch. } \\
\text { (127151) }\end{array}$ & Shwawan & Fruit, Leaves & September & Forests & $\begin{array}{l}\text { Women, } \\
\text { men, kids }\end{array}$ & $\begin{array}{l}\text { The fruits are directly consumed while the fresh leaves } \\
\text { are used as tea. }\end{array}$ & 1.0 & Yes \\
\hline
\end{tabular}


Table 3 Folk uses of wild food plants recorded in the study area (Continued)

\begin{tabular}{|c|c|c|c|c|c|c|c|c|}
\hline \multicolumn{9}{|l|}{ Oleaceae } \\
\hline Oxalis corniculata L. (127198) & Therwashka & Leaves & May & Waste land & Kids & Directly consumed. & 0.06 & Yes \\
\hline \multicolumn{9}{|l|}{ Oxalidaceae } \\
\hline Periploca aphylla Decne. (127156) & \multirow[t]{2}{*}{ Barrarr } & \multirow{2}{*}{$\begin{array}{l}\text { Fruit and } \\
\text { young } \\
\text { stems }\end{array}$} & \multirow[t]{2}{*}{ May } & \multirow[t]{2}{*}{ Waste land } & \multirow[t]{2}{*}{ Kids } & \multirow{2}{*}{$\begin{array}{l}\text { The fresh green fruits and young stems are chewed and } \\
\text { the sweet tasting sap is swallowed. }\end{array}$} & \multirow[t]{2}{*}{0.06} & \multirow[t]{2}{*}{ Yes } \\
\hline Apocynaceae & & & & & & & & \\
\hline Periploca hydaspidis Falc. (127128) & Khwaza waley & Fruit & May & Barren land & Kids & Directly consumed. & 0.01 & Yes \\
\hline \multicolumn{9}{|l|}{ Apocynaceae } \\
\hline Physalis divaricata D. Don (127181) & \multirow{2}{*}{$\begin{array}{l}\text { Band } \\
\text { malkhovj }\end{array}$} & \multirow[t]{2}{*}{ Fruit } & \multirow[t]{2}{*}{ August } & \multirow[t]{2}{*}{ Waste land } & \multirow[t]{2}{*}{ Kids } & \multirow[t]{2}{*}{ Directly consumed. } & \multirow[t]{2}{*}{0.01} & \multirow[t]{2}{*}{ No } \\
\hline Solanaceae & & & & & & & & \\
\hline $\begin{array}{l}\text { Pinus gerardiana Wall. ex D.Don } \\
\text { (127203) }\end{array}$ & Zanghozai & Fruit & October & Pine forests & Men, kids & Directly consumed. & 0.59 & Yes \\
\hline \multicolumn{9}{|l|}{ Pinaceae } \\
\hline $\begin{array}{l}\text { Pinus wallichiana A.B.Jacks. } \\
\text { (127213) }\end{array}$ & Nashtar & Fruit & October & Pine forests & Men, kids & Directly consumed. & 0.29 & Yes \\
\hline \multicolumn{9}{|l|}{ Pinaceae } \\
\hline Anacardiaceae & Shrawan & Fruit & October & Forests & $\begin{array}{l}\text { Women, } \\
\text { men, kids }\end{array}$ & Directly consumed. & 0.30 & Yes \\
\hline Pistacia khinjuk Stocks (127259) & Saho shrawan & Fruit & October & Forests & Men, kids & Directly consumed. & 0.01 & Yes \\
\hline \multicolumn{9}{|l|}{ Anacardiaceae } \\
\hline Portulaca quadrifida L. (127257) & Pakharai & Aerial parts & May-July & Waste land & Women & Young fresh leaves including young shoots are boiled in & 0.04 & Yes \\
\hline Portulacaceae & & & & & & $\begin{array}{l}\text { the leaves are moved to another container containing } \\
\text { ghee with fried onions \& red chilies. Kept on the fire until } \\
\text { water is evaporated \& ghee appears on top. This is then } \\
\text { eaten with bread. }\end{array}$ & & \\
\hline Punica granatum L. (127208) & Nargos & Fruit & September & Mountains \& & $\begin{array}{l}\text { Women, } \\
\text { men kids }\end{array}$ & Directly consumed. & 0.55 & Yes \\
\hline Lythraceae & & & & & & & & \\
\hline Pyracantha M. Roam (127127) & Khra sharavo & Fruit & September & Mountain & Men, kids & Directly consumed. & 0.01 & Yes \\
\hline Rosaceae & & & & for & & & & \\
\hline $\begin{array}{l}\text { Ribes alpestre Wall. ex Decne. } \\
(127193)\end{array}$ & Sheen korai & Fruit & July & $\begin{array}{l}\text { Bushy } \\
\text { vegetation }\end{array}$ & Kids & Directly consumed. & 0.01 & Yes \\
\hline Grossulariaceae & & & & & & & & \\
\hline Rosaceae & & & & & & & & \\
\hline Rubus ulmifolius Schott (127197) & Gharangavo & Fruit & August & Fertile & Men, kids & Directly consumed. & 0.26 & Yes \\
\hline Rosaceae & & & & valleys & & & & \\
\hline
\end{tabular}


Table 3 Folk uses of wild food plants recorded in the study area (Continued)

\begin{tabular}{|c|c|c|c|c|c|c|c|c|}
\hline $\begin{array}{l}\text { Salvia moorcroftiana Wall. ex } \\
\text { Benth. (127131) }\end{array}$ & Dersai & Stem & May-June & Waste land & $\begin{array}{l}\text { Women, } \\
\text { men, kids }\end{array}$ & $\begin{array}{l}\text { The external green part of the semi-matured stem is } \\
\text { removed and the remaining white juicy part is eaten raw. }\end{array}$ & 0.47 & No \\
\hline \multicolumn{9}{|l|}{ Lamiaceae } \\
\hline $\begin{array}{l}\text { Sideroxylon mascatense (A.DC.) } \\
\text { T.D.Penn. (127185) }\end{array}$ & Guargur & Fruit & June & $\begin{array}{l}\text { Low land } \\
\text { forests }\end{array}$ & $\begin{array}{l}\text { Women, } \\
\text { men, kids }\end{array}$ & Directly consumed. & 0.55 & Yes \\
\hline \multicolumn{9}{|l|}{ Sapotaceae } \\
\hline $\begin{array}{l}\text { Solanum americanum Mill. } \\
\text { (127158) }\end{array}$ & Malkhovj & Fruit & June & Waste land & Kids & Directly consumed. & 0.09 & Yes \\
\hline \multicolumn{9}{|l|}{ Solanaceae } \\
\hline $\begin{array}{l}\text { Spiraea canescens D.Don (127265) } \\
\text { Rosaceae }\end{array}$ & Sra wany & $\begin{array}{l}\text { Fruit, stem, } \\
\text { bark }\end{array}$ & May & $\begin{array}{l}\text { Sloppy } \\
\text { \&shrubby } \\
\text { vegetation }\end{array}$ & Men, kids & $\begin{array}{l}\text { The fruits are directly consumed. The green stem cortex } \\
\text { is boiled in water with sugar which is then taken as tea }\end{array}$ & 0.12 & $\begin{array}{l}\text { Yes (fruit), } \\
\text { No (tea) }\end{array}$ \\
\hline $\begin{array}{l}\text { Thymus linearis Benth. (127211) } \\
\text { Lamiaceae }\end{array}$ & Marveiy & Leaves & $\begin{array}{l}\text { June- } \\
\text { September }\end{array}$ & Waste land & $\begin{array}{l}\text { Women, } \\
\text { men, kids }\end{array}$ & The leaves are used as herbal tea. & 0.09 & Yes \\
\hline $\begin{array}{l}\text { Tragopogon gracilis D.Don } \\
\text { (127167) }\end{array}$ & Shabey & Aerial parts & July & $\begin{array}{l}\text { Fertile valley } \\
\text { bottoms }\end{array}$ & Kids & Directly consumed. & 0.12 & No \\
\hline \multicolumn{9}{|l|}{ Asteraceae } \\
\hline $\begin{array}{l}\text { Tulipa lehmanniana Merckl. } \\
\text { (127148) }\end{array}$ & Sondai & Bulb & April-June & $\begin{array}{l}\text { Mountain } \\
\text { plateaus }\end{array}$ & Men, kids & Directly consumed. & 0.27 & Yes \\
\hline \multicolumn{9}{|l|}{ Liliaceae } \\
\hline $\begin{array}{l}\text { Viburnum cotinifolium D. Don } \\
\text { (127210) }\end{array}$ & Thorayi & Fruit & August & $\begin{array}{l}\text { Mountain } \\
\text { forests }\end{array}$ & $\begin{array}{l}\text { Women, } \\
\text { men, kids }\end{array}$ & Directly consumed. & 0.56 & Yes \\
\hline \multicolumn{9}{|l|}{ Adoxaceae } \\
\hline $\begin{array}{l}\text { Viscum cruciatum Sieber ex } \\
\text { Boiss. (127269) }\end{array}$ & $\begin{array}{l}\text { Da shwawna } \\
\text { lewanai }\end{array}$ & Leaves & May & $\begin{array}{l}\text { Parasite of olive } \\
\text { trees }\end{array}$ & $\begin{array}{l}\text { Women, } \\
\text { men }\end{array}$ & The leaves are used as tea. & 0.11 & No \\
\hline \multicolumn{9}{|l|}{ Santalaceae } \\
\hline $\begin{array}{l}\text { Vitis flexuosa Thunb. (127270) } \\
\text { Vitaceae }\end{array}$ & Malavo & Fruit & June & Hedgerows & $\begin{array}{l}\text { Women, } \\
\text { men, kids }\end{array}$ & Directly consumed. & 0.02 & Yes \\
\hline Ziziphus jujuba Mill. (127153) & Bera & Fruit & July & Low land plains & Men, kids & Directly consumed. & 0.13 & Yes \\
\hline \multicolumn{9}{|l|}{ Rhamnaceae } \\
\hline $\begin{array}{l}\text { Ziziphus nummularia (Burm.f.) } \\
\text { Wight \& Arn. (127271) }\end{array}$ & Karkanr & Fruit & July & Low land plains & Kids & Directly consumed. & 0.01 & Yes \\
\hline \multicolumn{9}{|l|}{ Rhamnaceae } \\
\hline $\begin{array}{l}\text { Ziziphus oxyphylla Edgew. (127199) } \\
\text { Rhamnaceae }\end{array}$ & Heilaneiy & Fruit & July & Forests & $\begin{array}{l}\text { Women, } \\
\text { men, kids }\end{array}$ & Directly consumed. & 0.28 & Yes \\
\hline
\end{tabular}


inhabitants which migrate between the foothills and the mountains are exposed to both types of edible floras and thus have more knowledge than the other villagers. The inter- and intra-cultural variations of traditional knowledge are quite helpful in understanding its dynamic nature and patterns of development [60]. Each informant mentioned an average of 17.5 species (11 wild fruits, 6 wild vegetables, and 1.5 wild tea species). Figure 3 depicts the disparities in knowledge of different age groups on the basis of 5 years age differences. The age group 41-45 was more knowledgeable as a result of firsthand experience. Age as an influencing factor on WFP knowledge has also been mentioned in the literature [61]. However, the decrease of knowledge exhibited by the +45 age groups was the result of a reduction in their direct involvement in the collection and consumption of WFPs. Interestingly, the 21-25 years age group showed good traditional knowledge which is a sign of traditional knowledge consistency in the area. These individuals are involved in livestock herding during which they not only eat WFPs, but also collect them for later consumption at home. Younger people with considerable knowledge of WFPs (as a result of similar behaviors) have also been reported by Uprety et al. [20] and others $[62,63]$. The minor folk knowledge retained by the age group 26-30 can be explained by the fact that these age group members sometimes left their villages and migrated for labor to towns or overseas to support their families and came back to the villages later. Studying individual ethnobotanical knowledge has the potential to add to the systematic understanding of humanity's most prevalent and earliest form of knowledge [60].

\section{WFPs storage and their perceived medicinal values}

The offseason availability of WFPs through drying and storage are valuable practices as they provide food stability and therefore food security throughout the year [64]. Inhabitants of the study area store $16 \%$ (8) of WFP species, including Allium sp. (Fig. 4), Olea ferruginea, and Sideroxylon mascatense for use during the winter season; therefore, these species have significantly higher FC values than non-storable species (Mann-Whitney- $U$ Test for independent samples, $p<0.0001)$. We found that $29 \%$ (14 spp.) were exploited for additional cultural uses, for example, as fodder (Grewia tenax, G. villosa, Celtis australis), fuel and timber (Pinus wallichiana, $P$. gerardiana), as well as for multiple purposes (Olea ferruginea and Sideroxylon mascatense). The additional qualities of WFPs enhance their local preference as significantly higher FC values were recorded for such species (Mann-Whitney- $U$-Test for independent samples, $p=0.016)$. Furthermore, significantly higher FC values were also obtained for species with medicinal uses (Mann-Whitney- $U$-Test for independent samples, $p=0.001)$. The present results follow the general rule that the more versatile a plant, the more widespread its usefulness [65]. The medicinal importance of WFPs (given their role in the prevention of illnesses) has been explained in detail by $[3,15]$. The manifold uses of these plants demonstrate their local importance for subsistence and as cultural heritage [66, 67].

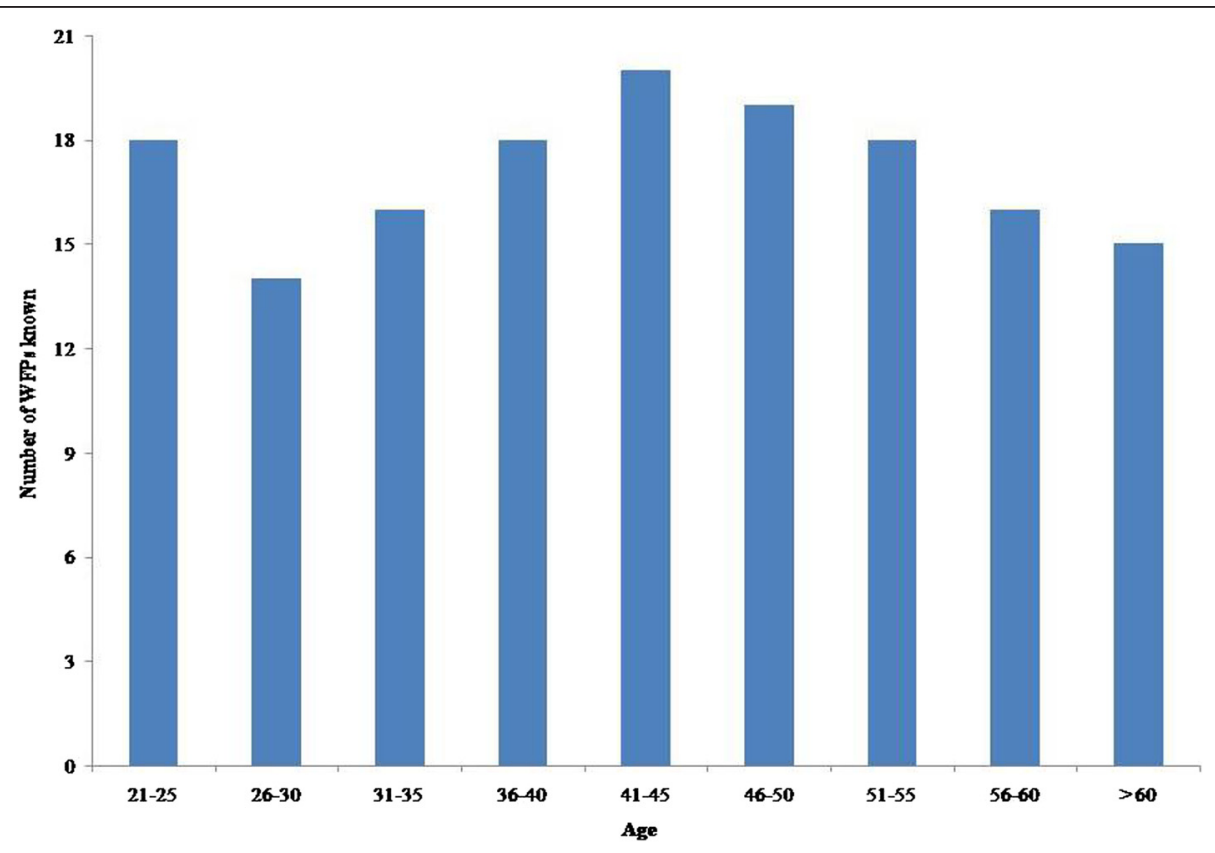

Fig. 3 Inter-generational variation of folk wild food plant knowledge recorded in the study area 


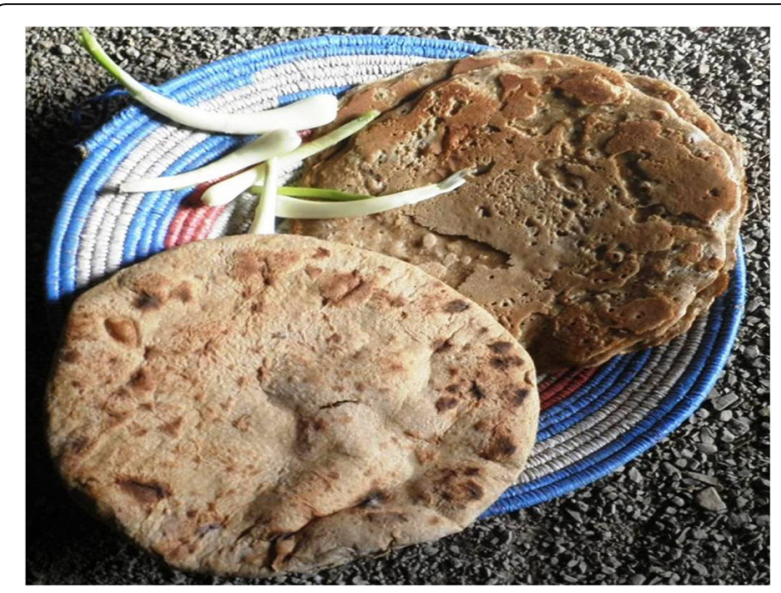

Fig. 4 Wild Allium sp. eaten with bread in a traditional lunch

\section{Trade, domestication, and conservation of WFPs}

In our documentation of WFPs, we observed that 14 $\%$ (7species) were sold in markets. The species with marketable potential, such as the species Pinus gerardiana, Caralluma tuberculate, and Ziziphus jujube (Fig. 5) were more important in the minds of local inhabitants (MannWhitney- $U$-Test for independent samples, $p=0.002$ ), as they provide opportunities for supplementing family income and such species have attracted worldwide attention [12]. Among all WFPs, 16 \% (8 species) were rarely available in the study area. The unavailability of these species was generally due to the unsuitable edaphic and climatic conditions (Lactuca dissecta, Notholirion thomsonianum, Pistacia khinjuk), although over-exploitation for marketing purposes was also responsible (Caralluma tuberculata). Sustainable use of such economically important species, however, could make a valuable contribution to the income

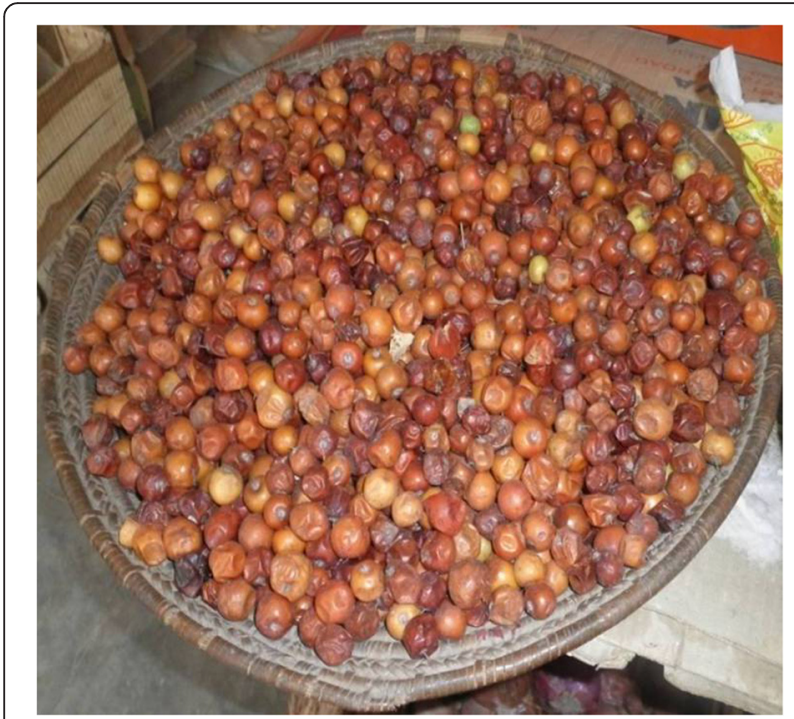

Fig. 5 Wild Ziziphus jujuba sold by local venders in the study area of indigenous communities [68, 69]. Additionally, it is believed that food botanical studies are vital for the conservation of cultural history, local identity and tradition dishes of a region [27]. The importance of traditional ecological knowledge in the conservation of biodiversity has been recognized by the United Nations Convention on Biological Diversity that calls for the recognition and protection of traditional knowledge [70]. In this study, $14 \%$ (7 species) of WFPs were semi-cultivated (i.e. present in both wild and cultivated forms), including Ficus palmata and Punica granatum. The domestication of species is the most important cultural development in the past 13,000 years of human history [14]. The wild species of Allium, Punica granatum, Cicer nuristanicum and Ficus palmata collected from the present study area may be quite useful for genetic crossover with their domesticated sister species in order to not only improve crop yields, nutrient levels, disease resistance, and the ability to withstand adverse climate change, but also broaden the genetic diversity of present crops to fulfill the requirements of the 21st Century [10, 11, 71].

\section{Seasonal variations in the ecological availability of WFPs}

The seasonality patterns of WFPs demonstrate that May, June, July, and August are regarded as the peak months, during which 33 \% (17 spp.), 37 \% (19 spp.), $39 \%$ (20 spp.) and $25 \%$ (13 spp.) are obtained, respectively. Likewise, January, February, November, and December are included as off months in which no WFPs are obtained. For fruits and vegetables the peak months are July, October and June, in that order. Similarly, the duration of availability of fruits is generally about only a month whereas for vegetables it lasts 2-5 months (Table 3). Overall, the majority of the species are available during the summer, i.e. hot-dry season which correlates well with shortages of cultivated food resources. The same phenomenon has been reported by Campbe [72], who explained that people need supplementary foods during periods of food shortages. The supply chain in the area was short (except for Pinus gerardinana) with collectors/ wholesalers selling either to retailers or directly to consumers. The collectors/wholesalers (mostly young boys) were responsible for bringing the WFPs to the market with little or no additional costs, where retailers and consumers can purchase them. The same details are mentioned by Karaan et al. [73] in relation to the marketing and supply chain of indigenous fruit markets in Tanzania, Zimbabwe and Zambia.

\section{Cultural value of WFPs}

Multiple methods of data collection were used (as explained above) for understanding the characteristics of WFPs. Detailed interviews demonstrate that Pinus gerardiana is the most important species in the area; 
however its FC value does not convey its actual importance. The reason for this is that Pinus gerardiana is not merely a WFP in the minds of locals; it also makes a tremendous contribution to their livelihood. Similarly, Punica granatum was appreciated as a quite important fruit in detailed interviews but its FC value does not represent this. Punica granatum is regarded less as a wild plant and more as a cultivated one, which causes it to be less cited. Conversely, Notholirion thomsonianum was recorded with a high FC value (0.31) in freelisting but less attention was given during the detailed interviews. On the other hand, the interviews suggested that wild tea species have no importance in the area but its separate freelisting made it a category that highly influenced their FC values. Despite the importance of different quantitative tools, semi-structured, detailed interviews are the best way of exploring the actual importance of ethnobotanical species [65]. Reyes-García et al. [74] stated that the value obtained through a freelisting does not necessarily correspond to its practical value as they found that some species frequently mentioned in freelisting exercises were rarely used. Multiple methods have to be employed for determining the present uses of plants as different indices do not, in all cases, measure the plants' uses but rather their knowledge [65].

\section{Collection sites}

This study investigated foothill and mountain villages that were separated on the basis of altitude, flora, rainfall, diversity and density of vegetation. This study reports a significantly higher number of species $61 \%$ (31 spp.) in the foothills as compared to the mountains 39 \% (20 spp.); however, the FC values of mountain species were significantly higher than for those in the foothills. A large proportion of informants do not migrate to the mountains, and as a result they remain unaware of the edible flora of these areas. Previous studies have mentioned that convenience and experience make the immediate vicinity a favorable collection site [55]. Moreover, the use of wild plants is primarily based on the utilization of species belonging to the closest ecological environments [75, 76]. Two possible factors may account for the high FC values: the selection of the best species among the diverse flora in the mountains and more dependence on natural food entities due to the inaccessibility of markets. Furthermore, there was a prevailing local belief that the mountains have more spiritual potential than the foothills which may influence local inhabitants to give more preference to mountainous species. Weckerle et al. [55], while examining ritual species in the Hengduan Mountains of Southwest China, explained the local perception regarding the availability of the best quality ritual species in high mountains due to a strong association of the mountains with god.

\section{Comparison with Pakistani ethnobotanical data}

To the best of our knowledge, after an extensive literature survey [42, 47-53, 58, 77-131], $73 \%$ (37) species recorded in this study had previously been reported by various investigations in Pakistan, whereas 27 \% (14 spp.) are newly reported WFPs and thus represent an addition to the knowledge of the wild edible flora of the country. The average FC values of the newly reported WFP species were significantly lower than the overall FC, which indicates that they are not very important in the area. However, some species which do have high FC values and thus represent important WFPs, such as Salvia moorcroftiana (FC: 0.47), Notholirion thomsonianum (FC: 0.31) and Cotoneaster pruinosus (FC: 0.22 ), had not yet been reported for the country. Several studies reported the use of these species for different purposes in other areas, including the medicinal use of Salvia moorcroftiana [77, 78, 132, 133] and Notholirion thomsonianum [125]. This may probably be due to specific cultural knowledge of each community, a phenomena that has been reported in other studies [45, 75]. Some species such as Viscum cruciatum, Cicer nuristanicum and Berberis calliobotrys may not have been reported in other areas due to their restricted occurrence in the country [134]. The newly documented species were collected from diverse habitats ranging from waste land to pine forests, by different collectors (women, men, kids), and during different seasons (Table 3). Moreover, they represented a broad range of WFPs including wild fruits (Berberis calliobotrys, Cotoneaster pruinosus), wild culinary vegetables (Notholirion thomsonianum, Cynoglossum lanceolatum), wild salad greens (Cirsium arvense, Salvia moorcroftiana), wild tea species (Limonium cabulicum, Spiraea canescens), and wild relatives of cash crops (Cicer nuristanicum, Allium sp.). This information may broaden the existing wild food spectra and might provide insight into food diversification. In the context of the recent rapid decline in traditional knowledge across the globe [135-137], exploration of new WFPs is quite encouraging.

\section{Conclusions}

The present study indicates that WFPs still represent an important part of the local food culture in the Thakht-eSulaiman Hills of NW Pakistan. The knowledge of such species, although persistent, is declining. Exposure to wider ecological zones, the surrounding vegetation and age of the inhabitant were all factors influencing the extent of traditional knowledge of WFPs. In addition to food value, the supplementary qualities of WFPs such as medicinal potential, cultural uses, marketing and storage make them more important in the local culture but also predispose them to extensive exploitation. There is a large potential for the harvesting, domestication and marketing of WFPs in the area, and if done properly, they could be a source of cash income for locals. The 
wild relatives of the domesticated food species could help increase genetic diversity for crop improvement and yield, thus addressing the present demand of human food security. The ongoing process of domestication of wild species in the area is of the utmost importance not only for the interests of local communities but also for global food diversification.

\section{Competing interests}

AP is the Editor-in-Chief of the Journal of Ethnobiology and Ethnomedicine.

\section{Authors' contributions}

KA designed and carried out the entire study: he conducted the fieldwork, as well as the data analysis and he wrote the first draft of the manuscript; AP contributed to the rationale of the data analysis, paper structure, and the discussion. All authors read and approved the final manuscript.

\section{Acknowledgments}

The authors are thankful to the local informants for sharing their traditional knowledge on the food uses of wild plants.

\section{Author details}

'Department of Environmental Sciences, COMSATS Institute of Information Technology, Abbottabad, Pakistan. ${ }^{2}$ University of Gastronomic Sciences, Piazza Vittorio Emanuele 9, I-12060 Pollenzo, Italy.

Received: 11 February 2016 Accepted: 3 April 2016

Published online: 08 April 2016

\section{References}

1. Jain SK. Human aspects of plant diversity. Econ Bot. 2000:54:459-70.

2. Etkin NL.. Eating on the Wild Side. The Pharmacologic, Ecologic and Social Implications of Using Noncultigens. Tucson: University of Arizona Press; 1994.

3. Leonti M, Nebel S, Rivera D, Heinrich M. Wild gathered food plants in the European Mediterranean: a comparative analysis. Econ Bot. 2006;60:130-42.

4. Dansi A, Adjatin A, Adoukonou-Sagbadja H, Faladé V, Yedomonhan H, Odou D, Dossou B.. Traditional leafy vegetables and their use in the Benin Republic. Genet Resour Crop Evol. 2008;55:1239-56.

5. Lamien-Meda A, Lamien CE, Compaoré MM, Meda RN, Kiendrebeogo M, Zeba B, Millogo JF, Nacoulma OG. Polyphenol content and antioxidant activity of fourteen wild edible fruits from Burkina Faso. Molecules. 2008;13:581-94

6. Odhav B, Beekrum S, Akula US, Baijnath H. Preliminary assessment of nutritional value of traditional leafy vegetables in KwaZulu-Natal, South Africa. J Food Comp Anal. 2007:20:430-5.

7. Tardío J, Pardo-de-Santayana MA, Morales R. Ethnobotanical review of wild edible plants in Spain. Bot J Linnean Soc. 2006;152:27-71.

8. Romeo R, Vita A, Testolin R, Hofer T. Mapping the vulnerability of mountain peoples to food insecurity. Rome: FAO; 2015

9. Colin K, Luigi G. Back to the roots: wild genes for food security. Samara: The International Newsletter of the Partners of the Millennium Seed Bank Partnership. 2010;18/19:1-8.

10. Ali-Shtayeh MS, Jamous RM, Al-Shafie JH, Elgharabah WA, Kherfan FA, Qarariah KH, Isra'S K, Soos IM, Musleh AA, Isa BA, Herzallah HM, Khlaif RB, Aiash SM, Ghadah M Swaiti GM, Abuzahra MA, Haj-Ali MM, Saifi NA, Azem HK, Nasrallah HA. Traditional knowledge of wild edible plants used in Palestine (Northern West Bank): a comparative study. J Ethnobiol Ethnomed. 2008;4:13.

11. Termote C, Van Damme P, Djailo BD. Eating from the wild: Turumbu, Mbole and Bali traditional knowledge on non-cultivated edible plants, District Tshopo, DRCongo. Genet Resour Crop Evol. 2011:58:585-618.

12. Millennium Ecosystem Assessment (MEA). Ecosystems and human wellbeing: synthesis. Washington, DC: Island Press; 2005

13. Hobblink H. Biodiversity. What's at stake?'. Currents. 2004;35/36:18-21.

14. Diamond J. Evolution, consequences and future of plant and animal domestication. Nature. 2002;418:700-7.
15. Leonti M. The co-evolutionary perspective of the food-medicine continuum and wild gathered and cultivated vegetables. Genet Resour Crop Evol. 2012;59:1295-302

16. Carrera-Bastos P, Fontes-Villalba M, O'Keefe JH, Lindeberg S, Cordain L. The western diet and lifestyle and diseases of civilization. Res Rep Clin Cardiol. 2011;2:15-35

17. Mann NJ. Paleolithic nutrition: what can we learn from the past? Asia Pac J Clin Nutr. 2004;13:S17

18. Cordain L, Brand-Miller J, Eaton SB, Hill K, Mann N. The paradoxical nature of hunter-gatherer diets: meat-based, yet non-atherogenic. Eur J Clin Nutr. 2002:56:42-52.

19. Eaton SB. Paleolithic vs. modern diets- selected pathophysiological implications. Eur J Nutr. 2000;39:67-70.

20. Uprety Y, Poudel RC, Shrestha KK, Rajbhandary S, Tiwari NN, Shrestha UB, Asselin H. Diversity of use and local knowledge of wild edible plant resources in Nepal. J Ethnobiol Ethnomed. 2012;8:16

21. Shinwari ZK, Nasim A. Ethnobotany in Pakistan. In: Selin H (ed.). Encyclopaedia of the History of Science, Technology, and Medicine in NonWestern Cultures. Netherlands: Springer; 2008. p. 1-14.

22. Govt. of Pakistan (GoP). Economic survey of Pakistan: 2005-2006. Islamabad: Ministry of Finance; 2007.

23. Stewart RR. An annotated catalogue of the vascular plants of West Pakistan and Kashmir. In: Nasir E, Ali Sl, editors. Flora of West Pakistan. Karachi: Fakhri Press; 1972. p. 1028

24. Munir H, Ejaz Q. Global water crisis and future food security in an era of climate change. Food Policy. 2010;35:365-77.

25. National Nutrition Survey (NNS). Agha Khan University, Pakistan Medical Research Council and Nutrition Wing, Cabinet Division, Government of Pakistan. 2011.

26. World Health Organization (WHO). Nutrition country profile: Pakistan. 2010. http://www.fao.org/ag/agn/nutrition/pak_en.stm (Accessed 09 Feb 2015).

27. Schunko C, Vogl CR. Organic farmers use of wild food plants and fungi in a hilly area in Styria (Austria). J Ethnobiol Ethnomed. 2010;6:17.

28. Ahmad K. Ethnobotanical studies of F.R, D. I. Khan, FATA, Pakistan. MSC Thesis, Gomal University, Pakistan; 2005

29. Raziq A, de Verdier K, Younas M. Ethnoveterinary treatments by dromedary camel herders in the Suleiman Mountainous Region in Pakistan: an observation and questionnaire study. J Ethnobiol Ethnomed. 2010;6:16.

30. Muhammad D. Country pasture/forage resource profiles. Pakistan: FAO Agriculture Department, Grassland and Pasture Crops Section; 2006. http:// www.fao.org/ag/agp/agpc/doc/counprof/PDF\%20files/Pakistan.pdf (Accessed 31 Dec 2015).

31. Bernard HR. Research Methods in Anthropology. Qualitative and Quantitative Approaches. Altamira, Walnut Creek, CA, USA; 2002.

32. Berlin EA, Berlin B. Some field methods in medical ethnobiology. Field Method. 2005;17:235-68

33. Brewer DD. Supplementary interviewing techniques to maximize output in freelisting tasks. Field Method. 2002;14:108-18.

34. Quinlan M. Considerations for collecting freelists in the field: examples from ethnobotany. Field Method. 2005;17:1-16.

35. Brewer DD. Cognitive indicators of knowledge in semantic domains. J Quant Anthropol. 1995;5:107-28.

36. International Society of Ethnobiology (ISE). International Society of Ethnobiology Code of Ethics. 2008. http://ethnobiology.net/code-of-ethics/ (Accessed 0101 2016).

37. Martin GJ. Ethnobotany. A methods manual. London: Chapman and Hall; 2004

38. Nasir E, Ali SI. Flora of West Pakistan Department of Botany. Karachi: University of Karachi; 1971-1995.

39. Ali SI, Qaisar M. Flora of Pakistan. Islamabad: Pakistan Agricultural Research Council; 1995-2010.

40. The Angiosperm Phylogeny Group (APG III). An update of the Angiosperm Phylogeny Group classification for the orders and families of flowering plants: APG III. Bot J Linnean Soc. 2009;161:105-21.

41. The Plant List. Version 1. 2010. http://www.theplantlist.org/ (Accessed 14 Nov 2014).

42. Abbasi AM, Khan MA, Khan N, Shah MH. Ethnobotanical survey of medicinally important wild edible fruits species used by tribal communities of Lesser Himalayas-Pakistan. J Ethnopharmacol. 2013;148:528-36.

43. Dogan Y, Ugulu I, Durkan N. Wild edible plants sold in the local markets of Izmir, Turkey Pak J Bot. 2013;45:177-84. 
44. Pieroni A. Evaluation of the cultural significance of wild food botanicals traditionally consumed in Northwestern Tuscany. Italy J Ethnobiol. 2001;21:89-104.

45. Pardo-de-Santayana M, Tardío J, Blanco E, Carvalho AM, Lastra JJ, San Miguel E, Morales R. Traditional knowledge of wild edible plants used in the northwest of the Iberian Peninsula (Spain and Portugal): a comparative study. J Ethnobiol Ethnomed. 2007;3:27.

46. Xu Y-K, Tao G-D, Liu H-M, Yan K-L, Dao X-S. Wild vegetable resources and market survey in Xishuangbanna, Southwest China. Econ Bot. 2004; 58:647-67.

47. Khan A, Gilani SS, Hussain F, Durrani MJ. Ethnobotany of Gokand Valley, District Buner, Pakistan. Pak J Biol Sci. 2003;6:362-9.

48. Latif A, Shinwari ZK. Sustainable market development for non timber forest products in Pakistan. Ethnobot Leaflets. 2005;2005:3.

49. Ali H, Qaiser M. The ethnobotany of Chitral valley, Pakistan with particular reference to medicinal plants. Pak J Bot. 2009;41:2009-41.

50. Awan MR, Shah M, Akber G, Ahmad S. Traditional uses of economically important plants of Chitral District, Malakand Division, NWFP, Pakistan. Pak J Bot. 2001;33:587-98.

51. Awan MR, Iqbal Z, Shah SM, Jamal Z, Jan G, Afzal M, Majid A, Gul A. Studies on traditional knowledge of economically important plants of Kaghan Valley, Mansehra District, Pakistan. J Med Plants Res. 2011;5:3958-67.

52. Khan M, Hussain F, Musharaf S. Ethnobotanical profile of Tehsil Takht-eNasratti, District Karak, Pakistan. J Med Plants Res. 2013;7:1636-51.

53. Murad W, Azizullah A, Adnan M, Tariq A, Khan KU, Waheed S, Ahmad A. Ethnobotanical assessment of plant resources of Banda Daud Shah, District Karak, Pakistan. J Ethnobiol Ethnomed. 2013;9:77.

54. Abbasi AM, Khan MA, Shah MH, Shah MM, Pervez A, Ahmad M. Ethnobotanical appraisal and cultural values of medicinally important wild edible vegetables of Lesser Himalayas-Pakistan. J Ethnobiol Ethnomed. 2013;9:66.

55. Weckerle CS, Huber FK, Yang YP, Sun WB. Plant knowledge of the Shuhi in the Hengduan Mountains, Southwest China. Econ Bot. 2006;60:3-23.

56. Nascimento VT, Lucena RFP, Maciel MIS, Albuquerque UP. Knowledge and use of wild food plants in areas of dry seasonal forests in Brazil. Ecol Food Nutr. 2013;52:317-43.

57. Mahapatra AK, Mishra S, Basak UC, Panda PC. Nutrient analysis of some selected wild edible fruits of deciduous forests of India: an explorative study towards non conventional bio-nutrition. Adv J Food Sci Technol. 2012;4:15-21.

58. Sher H, Elyemeni M, Sher H, Hussain K. Ethnobotanical and economic observations of some plant resources from the Northern parts of Pakistan. Ethnobot Res Appl. 2011;9:27-41.

59. Maundu PM. The status of traditional vegetable utilization in Kenya. In: Traditional African Vegetables. Proceedings of the IPGRI International workshop on Genetic Resources of Traditional Vegetables in Africa. Conservation and Use. Nairobi: ICRAF-HQ; 1997. p. 66-71.

60. Reyes-García V, Marti N, Mcdade T, Tanner S, Vadez V. Concepts and methods in studies measuring individual ethnobotanical knowledge. Ethnobiol. 2007;27:182-203.

61. Mengistu F, Hager H. Wild edible fruit species cultural domain, informan species competence and preference in three districts of Amhara region, Ethiopia. Ethnobot Res Appl. 2008;6:487-502.

62. Setalaphruk C, Price LL. Children's traditional ecological knowledge of wild food resources: a case study in a rural village in Northeast Thailand. J Ethnobiol Ethnomed. 2007;3:33.

63. Łuczaj $Ł$. Archival data on wild food plants used in Poland in 1948. J Ethnobiol Ethnomed. 2008;4:4.

64. Bvenura C, Afolayan AJ. The role of wild vegetables in household food security in South Africa: A review. Food Res Int. 2015;76:1001-11.

65. Tardío J, Pardo-de-Santayana M. Cultural importance indices: a comparative analysis based on the useful wild plants of Southern Cantabria (Northern Spain). Econ Bot. 2008:62:24-39.

66. Shrestha PM, Dhillion SS. Diversity and traditional knowledge concerning wild food species in a locally managed forest in Nepal. Agroforest Syst. 2006:66:55-63.

67. Ju Y, Zhuo J, Liu B, Long C. Eating from the wild: diversity of wild edible plants used by Tibetans in Shangri-la region, Yunnan. China J Ethnobiol Ethnomed. 2013;9:28.

68. FAO. Non wood forest products for rural income and sustainable forestry. Rome: FAO; 1995.

69. Agrawal A, Cashore B, Hardin R, Shepherd G, Benson C, Miller D. Background paper prepared for the United Nations Forum on Forests. 2013. http://www.un.org/esa/forests/pdf/session_documents/unff10/ EcoContrForests.pdf (Accessed 03 Jan 2016).

70. United Nations Environment Programme (UNEP). Traditional knowledge: indigenous and local communities knowledge, innovations and practices. Montreal: UNEP, Secretariat of the Convention on Biological Diversity; 2000. http://www.biodiv.org/doc/publications/guide.asp.

71. Hopkins JJ, Maxted N. Crop Wild Relatives: Plant conservation for food security. Peterborough: Natural England; 2011.

72. Campbe BM. The use of wild fruits in Zimbabwe. Econ Bot. 1987;41:375-85.

73. Karaan M, Ham C, Akinnifesi F, Moombe K, Jordaan D, Franzel S, Aithal A. Baseline marketing surveys and supply chain studies for indigenous fruit markets in Tanzania, Zimbabwe and Zambia. Nairobi: World Agroforestry Centre and CPWild Research Alliance; 2005.

74. Reyes-García V, Huanca T, Vadez V, Leonard W, Wilkie D. Cultural, practical, and economic value of wild plants: a quantitative study in the Bolivian Amazon. Econ Bot. 2006;60:62-74.

75. Ladio A, Lozada M, Weigandt M. Comparison of traditional wild plant knowledge between aboriginal communities inhabiting arid and forest environments in Patagonia, Argentina. J Arid Environ. 2007:69:695-715.

76. Salick J, Biun A, Martin G, Apin L, Beaman R. Whence useful plants? A direct relationship between biodiversity and useful plants among the Dusun of Mt. Kinabalu. Biodivers Conserv. 1999;8:797-818.

77. Barkatullah B, Ibrar M. Plants profile of Malakand Pass Hills, District Malakand, Pakistan. Afr J Biotechnol. 2013;10:16521-35

78. Shinwari Ml, Khan MA. Folk use of medicinal herbs of Margalla Hills National Park, Islamabad. J Ethnopharmacol. 2000;69:45-56.

79. Qureshi SJ, Khan MA. Ethnobotanical study of Kahuta from Rawalpindi District Pakistan. Online J Biol Sci. 2001;1(1):27-30.

80. Saghir IA, Awan AA, Majid S. Ethnobotanical Studies of Chikar and its Allied Areas of District Muzaffarabad. Online J Biol Sci. 2001;1:1165-70.

81. Iqbal I, Hamayun M. Studies on the traditional uses of plants of Malam Jabba valley, District Swat, Pakistan. Ethnobot Leaflets. 2004;2004:15.

82. Sher H, Khan ZD, Khan AU, Hussain F, Pei SJ. Ethnobotanical study on some plants in village Tigdari, District Swat, Pakistan. Acta Bot Yunnanica. 2004;10:42-54.

83. Baart $J$, Baart-Bremer EL, Sagar MZ. Names of Plants in Kalam Kohistani (Pakistan). Work Papers of the Summer Institute of Linguistics, University of North Dakota Session. 2004;48:1-24

84. Qureshi R. Floristic and ethnobotanical study of Desert-Nara Region, Sindh, Doctoral dissertation. Khairpur: Shah Abdul Latif University; 2004. http://prr. hec.gov.pk/Thesis/314.pdf (Accessed 01 Jun 2016).

85. Saqib Z, Sultan A. Ethnobotany of Palas valley, Pakistan. Ethnobot Leaflets. 2005;2005:28.

86. Hamayun M, Sumera AK, llyas I, Gohar R, Tariq H, Mir AK. Ethnobotanical profile of Utror and Gabral valleys, District Swat, Pakistan. Ethnobot Leaflets. 2005;2005:9.

87. Ahmad H, Samiullah K, Ahmad K, Muhammad H. Ethnobotanical Resources of Manikhel Forests, Orakzai Tirah, Pakistan. Ethnobot Leaflets. 2005;2005:38.

88. Arshad M, Ahmad M. Ethnobotanical study of Galliyat for botanical demography and bio-ecological diversification. Ethnobot Leaflets. 2005; 2005:21.

89. Qureshi RA, Ahmad I, Ishtiaq M. Ethnobotanical and phytosociological studies of Tehsil Gujar Khan District Rawalpindi. Asian J Plant Sci. 2006;5:890-3.

90. Hussain M, Shah GM, Khan MA. Traditional medicinal and economic uses of Gymnosperms of Kaghan Valley, Pakistan. Ethnobot Leaflets. 2006;2006:7.

91. Panhwar AQ, Abro H. Ethnobotanical studies of Mahal Kohistan (Khirthar National Park). Pak J Bot. 2007;39:2301-15.

92. Khan SW, Khatoon S. Ethnobotanical studies on some useful herbs of Haramosh and Bugrote valleys in Gilgit, Northern Areas of Pakistan. Pak J Bot. 2008:40:43

93. Husain SZ, Malik RN, Javaid M, Bibi S. Ethonobotanical properties and uses of medicinal plants of Morgah biodiversity park, Rawalpindi. Pak J Bot. 2008; 40:1897-911.

94. Jabeen A, Khan MA, Ahmad M, Zafar M, Ahmad F. Indigenous uses of economically important flora of Margallah Hills National Park, Islamabad, Pakistan. Afr J Biotechnol. 2009;8:763-84.

95. Hussain T, Muhammad IC. A floristic description of flora and ethnobotany of Samahni Valley (AK), Pakistan. Ethnobot Leaflets. 2009;7:6.

96. Jamal Z. Biodiversity, ethnobotany and conservation status of the flora of Kaghan Valley Mansehra, NWFP Pakistan, Doctoral dissertation. Islamabad: Quaid-i-Azam University; 2009. http://prr.hec.gov.pk/Thesis/2106S.pdf (Accessed 01 Jun 2016). 
97. Qasim M, Gulzar S, Shinwari ZK, Aziz IR, Khan MA. Traditional ethnobotanical uses of halophytes from Hub, Balochistan. Pak J Bot. 2010;42:1543-51.

98. Sher H, Al-Yemeni MN, Sher H. Forest Resource utilization assessment for economic development of rural community, Northern parts of Pakistan. J Med Plants Res. 2010;4:1197-208.

99. Khan MA, Khan MA, Hussain M, Ghulam GM. An ethnobotanical inventory of Himalayan region Poonch valley Azad Kashmir (Pakistan). Ethnobot Res Appl. 2010;8:107-23.

100. Marwat SK, Rehman FU, Usman K, Khakwani AZ, Ghulam S, Anwar N, Sadiq M. Medico-ethnobotanical studies of edible wild fruit plants species from the flora of north western Pakistan (DI Khan district). J Med Plants Res. 2011;5:3679-86.

101. Mahmood AD, Riffat NM, Zabta KS, Aqeel M. Ethnobotanical survey of plants from Neelum, Azad Jammu and Kashmir, Pakistan. Pak J Bot. 2011:43:105-10.

102. Shinwari S, Qureshi R, Baydoun E. Ethnobotanical study of Kohat Pass (Pakistan). Pak J Bot. 2011:43:135-9.

103. Ahmad I, Ibrar M, Ali N. Ethnobotanical Study of Tehsil Kabal, Swat District, KPK, Pakistan. J Bot. 2011;368572.

104. Ali H, Sannai J, Sher H, Rashid A. Ethnobotanical profile of some plant resources in Malam Jabba Valley of Swat, Pakistan. J Med Plants Res. 2011;5: 4676-87.

105. Munir M, Khan MA, Ahmed M, Bano A, Ahmed SN, Tariq K, Tabassum S, Mukhtar T, Ambreen M, Bashir S. Foliar epidermal anatomy of some ethnobotanically important species of wild edible fruits of northern Pakistan. J Med Plants Res. 2011;5:5873-80.

106. Khan MA, Khan SA, Qureshi MA, Ahmed G, Khan MA, Hussain M, Ghulam GM. Ethnobotany of some useful plants of Poonch Valley Azad Kashmir. J Med Plants Res. 2011;5:6140-51.

107. Sher H, Al-Yemeni M. Economically and ecologically important plant communities in high altitude coniferous forest of Malam Jabba, Swat, Pakistan. Saudi J Biol Sci. 2011;18:53-61.

108. Sher Z, Khan Z, Hussain F. Ethnobotanical studies of some plants of Chagharzai Valley, District Buner, Pakistan. Pak J Bot. 2011:43:1445-52.

109. Dar ME, Cochard R, Shreshta RP, Ahmad S. Plant resource utilization by local inhabitants around Machiara National Park, Azad Kashmir, Pakistan. J Food Agric Environ. 2012;10:1139-48

110. Murad W, Ahmad A, Ishaq G, Khan MS, Khan AM, Ullah I, Khan I. Ethnobotanical studies on plant resources of Hazar Nao forest, District Malakand, Pakistan. Pak J Weed Sci Res. 2012;18:509-27.

111. Badshah L, Hussain F, Sher Z. An over view of people plant interaction in the rangeland of District Tank, Pakistan. J Med Plant Res. 2012;6:2820-6.

112. Abbasi AM, Khan MA, Shah MH, Shah. Ethnobotanical survey of medicinally important wild edible fruits species used by tribal communities of Lesser Himalayas-Pakistan. J Ethnopharmacol. 2013;148(2):528-536.

113. Abbasi AM, Khan MA, Zafar M. Ethno-medicinal assessment of some selected wild edible fruits and vegetables of Lesser-Himalayas, Pakistan. J Ethnopharmacol. 2013;45:215-22.

114. Bano A, Ayub M, Rashid S, Sultana S, Sadia H. Ethnobotany and conservation status of floral diversity of Himalayan range of Azad Jammu and Kashmir Pakistan. Pak J Bot. 2013:45:243-51.

115. Shad AA, Shah HU, Bakht J. Ethnobotanical assessment and nutritive potential of wild food plants. J Anim Plant Sci. 2013;23:92-7.

116. Rehman S, Wazir SM, Khan RU, Khan SU, Farooq A. Ethnobotanically important plants of Humzoni, NWA, KPK, Pakistan. Int J Herb Med. 2013;1: 89-101.

117. Ishtiaq M, Maqbool M, Hussain T, Shah A. Role of indigenous knowledge in biodiversity conservation of an area: a case study on tree ethnobotany of Soona Valley, District Bhimber Azad Kashmir, Pakistan. Pak J Bot. 2013;45: 157-64.

118. Khan T, Khan IA, Rehman A, Ali H. Ethnobatanical studies on non-medicinal plants of Shinaki Valley Hunza, Gilgit-Baltistan. Int J Biol Sci. 2013;3:63-70

119. Ahmad K, Ahmad M, Weckerle C. Ethnobotanical studies of the eastern plains of Takht-e-Sulaiman hills. Pak J Bot. 2013;45:197-205.

120. Arshad M, Ahmad M, Ahmed E, Saboor A, Abbas A, Sadiq S. An ethnobiological study in Kala Chitta hills of Pothwar region, Pakistan: multinomial logit specification. J Ethnobiol Ethnomed. 2014;10:13.

121. Khan M, Shinwari ZK, Shah M, Musharaf S. An ethnobotanical survey of medicinal and other useful plants of Khattak tribe in Tehsil Karak, Khyber Pakhtunkhawa, Pakistan. J Med Plant Res. 2014;14:4.
122. Khan M, Hussain S. Diversity of wild edible plants and flowering Phenology of District Poonch (J\&K) in the NorthWest Himalaya. Indian J Sci Res. 2014;9: 032-8.

123. Ajaib M, Zaheer-UD-Din KH. Ethnobotanical studies of useful trees of District Kotli, Azad Jammu and Kashmir. Biologia Pak. 2014;60:63-71.

124. Sher Z, Hussain F, Ibrar M. Traditional knowledge on plant resources of Ashezai and Salarzai Valleys, District Buner, Pakistan. Afr J Plant Sci. 2014;8: 42-53.

125. Ajaib M, Haider SK, Zikrea A, Siddiqui MF. Ethnobotanical studies of shrubs and trees of Agra Valley Parachinar, Upper Kurram Agency, Pakistan. FUUAST J Biol. 2014:4:73.

126. Abbasi AM, Shah MH, Khan MA. Wild Edible Vegetables of Lesser Himalayas. New York: Springer; 2015. Volume 1.

127. Abbasi AM, Shah MH, Li T, Fu X, Guo X, Liu RH. Ethnomedicinal values, phenolic contents and antioxidant properties of wild culinary vegetables. J Ethnopharmacol. 2015;162:333-45.

128. Amjad MS, Arshad M, Qureshi R. Ethnobotanical inventory and folk uses of indigenous plants from Pir Nasoora National Park, Azad Jammu and Kashmir. Asian Pac J Trop Biomed. 2015;5:234-41.

129. Khan MP, Ahmad M, Zafar M, Sultana S, Ali MI, Sun H. Ethnomedicinal uses of Edible Wild Fruits (EWFs) in Swat Valley, Northern Pakistan. J Ethnopharmacol. 2015:173:191-203.

130. Khan MP, Ahmad M. Traditional preference of Wild Edible Fruits (WEFs) for digestive disorders (DDs) among the indigenous communities of Swat Valley-Pakistan. J Ethnopharmacol. 2015;174:339-54.

131. Amjad MS. Ethnobotanical profiling and floristic diversity of Bana Valley, Kotli (Azad Jammu and Kashmir), Pakistan. Asian Pac J Trop Biomed. 2015;5:292-9.

132. Abbasi AM, Khan MA, Ahmad M, Zafar M, Jahan S, Sultana S. Ethnopharmacological application of medicinal plants to cure skin diseases and in folk cosmetics among the tribal communities of North-West Frontier Province, Pakistan. J Ethnopharmacol. 2010;128:322-35.

133. Abbasi AM, Khan MA, Ahmed M, Zafar M. Herbal medicines used to cure various ailments by the inhabitants of Abbottabad District, North West Frontier Province, Pakistan. Indian J Trad it Know. 2010;9:175-83.

134. Tropicos/Flora of Pakistan. Pakistan Plant Database. http://www.tropicos.org/ Project/Pakistan (Accessed 01 Sep 2016).

135. Cox PA. Will tribal knowledge survive the millennium? Science. 2000:287:44-5.

136. Ramirez CR. Ethnobotany and the loss of traditional knowledge in the $21^{\text {st }}$ century. Ethnobot Res Appl. 2007:5:245-7.

137. McCarter J, Gavin MC. Local perceptions of changes in traditional ecological knowledge: a case study from Malekula Island, Vanuatu. Ambio. 2014:1:43:288-96.

\section{Submit your next manuscript to BioMed Central and we will help you at every step:}

- We accept pre-submission inquiries

- Our selector tool helps you to find the most relevant journal

- We provide round the clock customer support

- Convenient online submission

- Thorough peer review

- Inclusion in PubMed and all major indexing services

- Maximum visibility for your research

Submit your manuscript at www.biomedcentral.com/submit 